\title{
New Contribution to Faraday and Kerr Magneto-Optical Effects in Ferromagnets
}

\author{
A.V. VedyayeV ${ }^{a}$, M.Ye. ZHURAVleV $^{b, c, *}$ AND A.V. LOBACHEV ${ }^{a}$ \\ ${ }^{a}$ Faculty of Physics, Lomonosov Moscow State University, Leninskiye Gory 1, 119899 Moscow, Russia \\ ${ }^{b}$ Faculty of Liberal Arts and Sciences, St Petersburg State University, \\ 58-60 Galernaya St., 190000 St. Petersburg, Russia
}

${ }^{c}$ Kurnakov Institute of General and Inorganic Chemistry of RAS, 31 Leninsky prospect, 119991 Moscow, Russia

\begin{abstract}
We consider a new magneto-optic effect based upon spin-orbit interaction of the conducting electrons in ferromagnetic metal with the electric field of plane polarized light wave. We calculated off-diagonal components of conductivity tensor for this mechanism of a.c. conductivity. These components determine the current induced by the light wave in the direction perpendicular to the plane of the light polarization. Numerical evaluation shows that the conductivity for this new effect exceeds the off-diagonal components of conductivity tensor for ordinary magneto-optic effect. The components appear to be still considerably smaller than the diagonal elements of the conductivity tensor.
\end{abstract}

DOI: 10.12693/APhysPolA.133.523

PACS/topics: 78.20.Ls

\section{Introduction}

The first magneto-optical effects were discovered more than a hundred years ago. Nevertheless, they still attract great attention. Magneto-optical experiments can be arranged for the transmitted wave (i.e., Faraday magneto-optical effects) as well as reflected wave (i.e., Kerr magneto-optical effects) [1, 2]. Both the magnetic sample and the non-magnetic material in external magnetic field demonstrate magneto-optical response. Also, the magneto-optical effects differ by the mutual orientation of the polarization wave and magnetization of the sample. These effects serve as a tool for the investigation of electron band structure and details of magnetic state of metals and semiconductors. Magnetic aspects of bulk as well as surface electron structure can be investigated with magneto-optic experiments. Depending on the geometry of the experiment, the reflection or transmission of light through magnetized sample results in the change of the light polarization and intensity, in particular, the rotation of polarization vector of linearly polarized light or appearance of the ellipticity can take place.

The field of magneto-optical research is growing steadily. Whereas the first experiments were carried out in optical range, now infrared range as well as x-ray range are used in magneto-optical research. The new materials that demonstrated unusual properties or new functional potentials have become the subject of magneto-optical studies. In particular, magnetic nanocomposites and the multilayered films were investigated and the theory of magneto-optical effects in multilayered structures was developed $[3,4]$. Magneto-optical response of thin films of

*corresponding author; e-mail: myezhur@gmail.com topological insulators [5] and graphene were studied [6]. Comparatively new branch of magneto-optical research is the development of magneto-optical microstructure devices [7].

It is well known that magneto-optic phenomena require spin-orbit coupling (SOC) [8]. The quantitative analysis of the magnet-optical effects is based on calculation of the off-diagonal elements of permittivity tensor or conductivity tensor which are connected by a linear relation. The corresponding theory was developed in a set of papers (see, e.g., $[9,10])$. As a rule, the "intrinsic" bulk spin-orbit coupling results in magneto-optical response of magnetic crystal. In the present investigation, we demonstrate that the SOC between the conducting electrons of ferromagnetic material and the electric field of the light can provide the basis for magneto-optical effects. We calculate the contribution into the conductivity tensor originating from this type of SOC. We follow the calculational scheme of [9].

\section{Model and calculation}

We describe the system of conducting electrons of ferromagnetic metals and electromagnetic wave in oneelectron approximation:

$$
H_{F M}=H_{0}+H^{\prime}+H_{S O}
$$

where we take into account the potential energy of an electron in the crystal and the exchange splitting of conducting band,

$$
H_{0}=\left(\begin{array}{cc}
-\frac{\hbar^{2} \Delta}{2 m}+V(\boldsymbol{r})-\frac{J}{2} & 0 \\
0 & -\frac{\hbar^{2} \Delta}{2 m}+V(\boldsymbol{r})+\frac{J}{2}
\end{array}\right)
$$

The wave functions of $(2), H_{0} \psi_{n}=E_{n}(\boldsymbol{k}) \psi_{n}$ are Bloch functions, $\psi_{n}(\boldsymbol{k}, \boldsymbol{r})=\left(1 / N^{1 / 2}\right) \mathrm{e}^{\mathrm{i} k \boldsymbol{r}} u_{n}(\boldsymbol{k}, \boldsymbol{r})$, where $n$ is the band number and $E_{n}(\boldsymbol{k})$ is the energy of the state. 
Interaction of an electron with electromagnetic wave includes two terms, the first one is proportional to the scalar product of vector potential of electromagnetic wave $\boldsymbol{A}$ and electron momentum $\boldsymbol{p}=-\mathrm{i} \hbar \nabla$, the second one is the SOC between the conducting electron and electric field of the wave,

$$
H_{S O}=g(\boldsymbol{\tau}(\boldsymbol{E} \times \nabla)), \quad g=-\mathrm{i} e \hbar^{2} / 4 m^{2} c^{2},
$$

where $\boldsymbol{\tau}$ is the vector consisting of Pauli matrices. In (3) $\boldsymbol{E}$ is the electric field of the wave. In the common theory instead of $\boldsymbol{E}$, the gradient of electron potential energy $V(\boldsymbol{r})$ is used. These contributions into conductivity tensor are additive, therefore we calculate the off-diagonal elements of the tensor for system (1)-(3). We consider the geometry of the system, when the electric vector of the harmonic wave, $\boldsymbol{E}_{0} \mathrm{e}^{\mathrm{i} \omega t}$ is perpendicular to the magnetization of the ferromagnetic sample.

Applying time-dependent perturbation theory to construct wave function of (1), we find the following expression for the off-diagonal element of conductivity $\left(\sigma_{x y}^{\uparrow \uparrow}\right)$ and polarizability $\left(\alpha_{x y}^{\uparrow \uparrow}\right)$ tensors in the first order in SOC:

$$
\begin{aligned}
\sigma_{x y}^{\uparrow \uparrow} & =\frac{\mathrm{e}^{2} \hbar^{2}}{16 \pi^{3} m^{3} c^{2}} \sum_{l \neq n} \int \frac{2 \omega_{n l}}{\omega_{n l}^{2}-\omega^{2}}\left|P_{n l}\right|^{2} \mathrm{~d} \boldsymbol{k}, \\
\alpha_{x y}^{\uparrow \uparrow} & =\frac{\mathrm{e}^{2} \hbar^{2}}{8 \pi^{2} m^{3} c^{2}} \int \frac{\delta\left(\omega_{n l}-\omega\right)}{\omega}\left|P_{n l}\right|^{2} \mathrm{~d} \boldsymbol{k},
\end{aligned}
$$

where

$$
\begin{aligned}
& f_{n l}^{\uparrow \uparrow}(\omega, t)= \\
& \left\{\frac{\left(1-\mathrm{e}^{-\mathrm{i}\left(\omega_{l}^{\uparrow}-\omega_{n}^{\uparrow}-\omega\right) t}\right)}{\omega_{l}^{\uparrow}-\omega_{n}^{\uparrow}-\omega}+\frac{\left(1-\mathrm{e}^{-\mathrm{i}\left(\omega_{l}^{\uparrow}-\omega_{n}^{\uparrow}+\omega\right) t}\right)}{\omega_{l}^{\uparrow}-\omega_{n}^{\uparrow}+\omega}\right\} .
\end{aligned}
$$

The summation in (4) is over the energy bands, factor $P_{l n}^{\uparrow \uparrow}(k)$ is a matrix element of derivative between Bloch functions $u_{n, \boldsymbol{k}}(\boldsymbol{r})$,

$$
P_{n l}^{\uparrow \uparrow}(k)=\int u_{l, k}^{\uparrow *}(r) \partial_{x} u_{n, k}^{\uparrow}(r) \mathrm{d} \tau_{0} .
$$

We use the notation $\int \ldots \mathrm{d} \tau_{0}$ for the integration over the unit cell. To obtain (4) and (5) we used the averaging proposed by Wilson in [11].

Total expression for off-diagonal element of the conductivity tensor is the difference of (4) and analogous expression for "spin-down" electrons. This difference is proportional to the magnetization. The matrix elements (7) are similar to those used by Argyres [9]. The essential difference between our expression for the off-diagonal conductivity and those of [9] is following. In [9] the interaction of an electron and the light is determined by $H^{\prime}=(e / m c) \boldsymbol{A}(\boldsymbol{r}, t) \cdot \boldsymbol{p}$, whereas in our mechanism this is spin-orbit interaction between an electron and electrical field of the electromagnetic wave. We do not need $H^{\prime}$ to get off-diagonal conductivity. Our expression for off-diagonal conductivity (4) does not depend on electromagnetic wave frequency $\omega$. The ratio of the calculated tensor element (4) to the one, calculated in [9] is

$$
\sim \frac{\omega m\left(E_{n}-E_{m}\right)}{2 \hbar(\operatorname{grad} V(\boldsymbol{r}))\left\langle m\left|\partial_{x}\right| n\right\rangle}
$$

Using the estimations for $\operatorname{grad} V(\boldsymbol{r})$ and matrix element

$$
\left\langle l\left|\partial_{x}\right| n\right\rangle=\int u_{l}^{*}(\boldsymbol{r}) \partial_{x} u_{n}(\boldsymbol{r}) \mathrm{d} \tau_{0}
$$

presented in [9], we found that the ratio (7) is of the order of unity for optical range. The total value of conductivity tensor element in the first order in SOC is the sum of (4) and the corresponding expression for "spin-down" electrons, which has opposite sign.

\section{Conclusion}

In conclusion, we have calculated the contribution into off-diagonal elements of conductivity tensor originated from the SOC between the conducting electrons of ferromagnetic crystal and the electrical field of the light. Numerical estimation shows that the obtained matrix element is of the same order as the matrix element calculated in the framework of standard theory [9] in optical range. The off-diagonal elements of conductivity and polarizability tensors determine the magneto-optical response of the crystal. Therefore, we propose new mechanism of magneto-optical Faraday and Kerr effects which does not depend on the bulk SOC of the crystal. The total magneto-optical response of magnetic crystal is determined by the sum of the contributions into off-diagonal conductivity and polarizability, originated from standard mechanism [9] and the mechanism based on SOC of the electrons with electromagnetic wave presented in our analysis. Correspondingly, complex indexes of refraction and the characteristics of magneto-optical effects now (Section 3 of Argyres article) are expressed through these sums. Expression (5) for the elements of polarizability tensor depends as $\omega^{-1}$ on light frequency, whereas the Argyres theory gives $\omega^{-2}$. This difference can be useful for the experimental test of the proposed theory. The semiconductors demonstrate comparable large values of polarizability. That is why they are more convenient for the experimental testing of the suggested mechanism than metals are.

\section{Acknowledgments}

The authors are grateful to E.A. Gan'shina for valuable discussion.

\section{References}

[1] P. Weinberger, Philosophical Magazine Letters 88, 897 (2008).

[2] H. Ebert, Rep. Prog. Phys. 59, 1665 (1996).

[3] A.B. Granovsky, I.V. Bykov, E.A. Gan'shina, V.S. Gushchin, M. Inoue, Yu.E. Kalinin, A.A. Kozlov, A.N. Yurasov, J. Exp. Theor. Phys. 96, 1104 (2003).

[4] M. Inoue, T. Fujii, J. Appl. Phys. 81, 5659 (1997). 
[5] W.-K. Tse, A.H. MacDonald, Phys. Rev. Lett. 105, 057401 (2010).

[6] L.A. Chizhova, J. Burgdörfer, F. Libisch, Phys. Rev. B 92, 125411 (2015).

[7] F. Fan, S. Chen, S.-J. Chang, IEEE J. Sel. Top. Quant. Electron. 23, 8500111 (2017).

[8] H.R. Hulme, Proc. Roy. Soc. (London) A 135, 237 (1932).
[9] P.N. Argyres, Phys. Rev. 97, 334 (1955).

[10] J. Zak, E.R. Moog, C. Liu, S.D. Bader, J. Magn. Magn. Mater. 89, 107 (1990).

[11] A.H. Wilson, Proc. Roy. Soc. A 151, 274 (1935). 